\title{
Hydrogen Peroxide in Inflammation: Messenger, Guide, and Assassin
}

\author{
C. Wittmann, ${ }^{1}$ P. Chockley, ${ }^{1}$ S. K. Singh, ${ }^{1}$ L. Pase, ${ }^{2}$ G. J. Lieschke, ${ }^{3}$ and C. Grabher ${ }^{1}$ \\ ${ }^{1}$ Institute of Toxicology and Genetics, Karlsruhe Institute of Technology (KIT), 76133 Karlsruhe, Germany \\ ${ }^{2}$ Cell Cycle and Cancer Genetics Laboratory, Peter MacCallum Cancer Centre, East Melbourne, VIC 3002, Australia \\ ${ }^{3}$ Australian Regenerative Medicine Institute, Monash University, Clayton, VIC 3800, Australia
}

Correspondence should be addressed to C. Grabher, clemens.grabher@kit.edu

Received 9 February 2012; Accepted 29 April 2012

Academic Editor: Christopher Hall

Copyright () 2012 C. Wittmann et al. This is an open access article distributed under the Creative Commons Attribution License, which permits unrestricted use, distribution, and reproduction in any medium, provided the original work is properly cited.

\begin{abstract}
Starting as a model for developmental genetics, embryology, and organogenesis, the zebrafish has become increasingly popular as a model organism for numerous areas of biology and biomedicine over the last decades. Within haematology, this includes studies on blood cell development and function and the intricate regulatory mechanisms within vertebrate immunity. Here, we review recent studies on the immediate mechanisms mounting an inflammatory response by in vivo analyses using the zebrafish. These recently revealed novel roles of the reactive oxygen species hydrogen peroxide that have changed our view on the initiation of a granulocytic inflammatory response.
\end{abstract}

\section{Introduction}

The innate immune system comprises the cells and mechanisms that defend the host from infection by other organisms or damage to tissue integrity, in a nonspecific manner. This means that the cells of the innate system recognise and respond to pathogens and trauma in a generic way, but unlike the adaptive immune system, it does not confer long-lasting or protective immunity to the host. The innate immune system provides an immediate defence. A typical vertebrate immune response depends on the orchestrated motility and activity of various haematopoietic compartments and their interactions that ultimately control the magnitude of the response [1-3]. Inflammation is one of the first responses of the immune system to infection or irritation. Stimulated by factors released from injured cells, it serves to establish a physical barrier against the spread of infection. This further promotes healing of any damaged tissue following the clearance of pathogens or cell debris. Molecules produced during inflammation sensitise pain receptors, cause localised vasodilatation of blood vessels, and attract phagocytes, especially neutrophils and macrophages, which then trigger other parts of the immune system.

Failure to initiate a response allows uncontrolled proliferation of invading microorganisms and severe tissue damage that may become fatal. Failure to resolve an immune response can also cause severe tissue damage, due to persistent degranulation, and may lead to chronic inflammation, which ceases to be beneficial to the host. Overall, inflammation is now recognised as a central feature of prevalent pathologies, such as atherosclerosis, cancer, asthma, thyroiditis, inflammatory bowel disease, autoimmune disease, as well as Alzheimer's and Parkinson's disease [4-6]. Hence, the regulation of an inflammatory response is an active field of research. New players or novel functions of old players continue to be identified and we are only beginning to understand their specific function at the corresponding level during inflammation. Hydrogen peroxide is an example of a molecule with a long known function for pathogen clearance in inflammation. Here, we discuss how recent work using the zebrafish model has revealed a pivotal role of hydrogen peroxide in mounting an inflammatory response.

\section{Cellular Lifecycle of Hydrogen Peroxide}

Hydrogen peroxide belongs to a group of chemically reactive molecules known as reactive oxygen species (ROS) that arise through oxidative metabolism. ROS comprise oxygen derived small molecules such as the oxygen radicals: 
superoxide, hydroxyl, peroxyl, and alkoxyl; or the nonradicals: hypochlorous acid, ozone, singlet oxygen, and the current topic in focus, hydrogen peroxide [7]. ROS generation can either occur as a by-product of cellular metabolism (e.g., in mitochondria through autoxidation of respiratory chain components) or it can be created by enzymes with the primary function of ROS generation [8]. Enzymes capable of rapidly increasing local $\mathrm{H}_{2} \mathrm{O}_{2}$ levels include the family of NADPH oxidases [7] and other oxidases such as xanthine oxidase [9] and 5-lipoxygenase [10]. The mammalian NADPH oxidase family encompasses 7 members, which are NOX1-5 and DUOX1-2. To date, a single isoform of duox and four nox genes (noxl, 2, 4, 5) have been identified in the zebrafish genome [11]. Each member is capable of converting $\mathrm{NADPH}$ to $\mathrm{NADP}^{+}$and then transporting the freed electrons across membranes. DUOX enzymes are capable of direct hydrogen peroxide production, while NOXes1-5 produce superoxide, which is rapidly converted to $\mathrm{H}_{2} \mathrm{O}_{2}$ by a separate superoxide dismutase or occurs spontaneously [12]. $\mathrm{H}_{2} \mathrm{O}_{2}$ may subsequently be utilised by peroxidase, such as thyroperoxidase, to produce thyroid hormones or myeloperoxidase and lactoperoxidase to generate more potent ROS. However, if not consumed, high concentrations of $\mathrm{H}_{2} \mathrm{O}_{2}$ may result in DNA damage and modifications of proteins, lipids and other molecules [13]. Thus, to avoid $\mathrm{H}_{2} \mathrm{O}_{2}$-mediated deleterious effects, excess $\mathrm{H}_{2} \mathrm{O}_{2}$ is usually rapidly catalysed or reduced by various antioxidant enzymes: such as glutathione peroxidase and catalase [14].

\section{Functional Activities of $\mathbf{H}_{2} \mathbf{O}_{2}$}

$\mathrm{H}_{2} \mathrm{O}_{2}$ is also involved in many regulatory cellular events including the activation of transcription factors, cell proliferation, and apoptosis [8]. $\mathrm{H}_{2} \mathrm{O}_{2}$ produced from the mitochondrial electron transport chain has been shown to play a role in haematopoietic cell differentiation and cell division in flies $[15,16]$. NADPH oxidase generated $\mathrm{H}_{2} \mathrm{O}_{2}$ can affect cardiac differentiation [17], vascularisation [18], and angiogenesis [19]. In targeting cysteine and methionine residues of protein kinases and phosphatases, $\mathrm{H}_{2} \mathrm{O}_{2}$ is capable of modulating a number of principal signalling cascades including ERK, JNK, p38, MAPK, and PI3K/Akt [20, 21].

\subsection{Inflammation-Related Functions}

3.1.1. Respiratory Burst. The classical physiological role attributed to $\mathrm{H}_{2} \mathrm{O}_{2}$ is its capability to induce bacterial killing [12]. NOX2 is the enzyme responsible for phagocyte respiratory burst responses and is expressed in neutrophils, eosinophils, monocytes/macrophages, as well as nonphagocytic cells such as fibroblasts, cardiomyocytes, haematopoietic stem cells, and endothelial cells [7]. Under resting conditions neutrophil NOX2 resides in secondary granules, which upon activation of neutrophils fuse with phagosomal as well as plasma membranes [22].

The NADPH-oxidase-mediated respiratory burst response of neutrophils generates two superoxide anions by transporting two electrons from one NADPH across the membrane to the extracellular or intra-phagosomal space. Superoxide is further converted into hydrogen peroxide either through spontaneous dismutation, which involves the consumption of two protons, or facilitated by the catalytic activity of superoxide dismutase. Hydrogen peroxide alone and in conjunction with the amplification activity of myeloperoxidase (MPO) is responsible for bacterial killing $[23,24]$. MPO, which is abundantly present in phagocyte granules, catalyses the conversion of halides and pseudohalides such as $\mathrm{Cl}^{-}, \mathrm{I}^{-}, \mathrm{Br}^{-}$, and $\mathrm{SCN}^{-}$to form hypohalous acids or pseudohypohalous acids. $\mathrm{HOCl}$, however, is the primary MPO product in neutrophils responsible for bacterial killing.

\subsubsection{Hydrogen Peroxide Mounting an Inflammatory Re-} sponse. Recent advances accomplished by utilising the model organism zebrafish greatly expanded our view of $\mathrm{H}_{2} \mathrm{O}_{2}$ mediated cellular activities. The optical transparency of zebrafish larvae offers the unique advantage of real-time monitoring an immune response in a whole animal context. This is in contrast to in vitro studies and/or end-point analyses of stained tissues. Additionally, a recently developed genetically encoded $\mathrm{H}_{2} \mathrm{O}_{2}$ sensor provided an elegant solution for investigating the role of hydrogen peroxide dynamics during an immune response in vivo [25].

The previous view on the critical mechanisms in immediate inflammation focused on the activity of damageassociated molecular patterns (DAMPs) and pathogen-associated molecular patterns (PAMPs). Tissue damage results in the release of intracellular DAMPs usually hidden from the immune system (i.e., ATP, uric acid, lipids, DNA, nuclear proteins) or extracellular DAMPs released through degradation of extracellular matrix upon tissue injury (i.e., hyaluronan, byglycan, heparan sulfate). The receiving cell senses these signals through 5 different types of pattern recognition receptors (PRRs). Activation of these receptors in turn activates downstream NFkB, MAPK, or type I interferon-signalling pathways that are important for inflammatory and antimicrobial responses. The significance of DAMPs, PAMPs, and PRRs is comprehensively reviewed elsewhere [26, 27]. However, the mechanisms for immediate immune cell recruitment were not well defined.

Recently, Niethammer et al. described for the first time that wounded epithelium of zebrafish larvae produces a tissue-scale gradient of $\mathrm{H}_{2} \mathrm{O}_{2}$ mediating leukocyte recruitment [28]. This finding was in contrast to the prevalent view that leukocytes undergoing an oxidative burst response were the only source of $\mathrm{H}_{2} \mathrm{O}_{2}$ at a site of trauma or infection [29]. The authors employed the genetically encoded ratiometric HyPer sensor to visualise $\mathrm{H}_{2} \mathrm{O}_{2}$ in vivo and in real time. HyPer consists of the bacterial $\mathrm{H}_{2} \mathrm{O}_{2}$-sensitive transcription factor, OxyR, fused to a circularly permutated yellow fluorescent protein (YFP). Cysteine oxidation of OxyR induces a conformational change in the YFP that increases emission excited at $500 \mathrm{~nm}$ and decreases emission excited at $420 \mathrm{~nm}$. This change is rapidly reversible within the reducing cytoplasmic environment, which allows dynamic monitoring of the intracellular hydrogen peroxide concentration [30]. 
Tailfin transection on zebrafish larvae induced a rapid increase in $\mathrm{H}_{2} \mathrm{O}_{2}$ levels extending approximately 100-200 $\mu \mathrm{m}$ from the wound margin as a decreasing concentration gradient. Furthermore, gradient formation preceded leukocyte arrival at the scene and $\mathrm{H}_{2} \mathrm{O}_{2}$ levels started to decrease again with accumulation of immune cells. Generation of the gradient as well as leukocyte recruitment was dependent on the activity of Duox in epithelial cells. Both, genetic knockdown of Duox and chemical inhibition of oxidase activity abolished gradient formation and significantly decreased absolute numbers of leukocytes at the wound margin, without affecting general cellular motility. These findings were corroborated by a study in drosophila focusing on prioritising competing signals by migrating macrophages [31] emphasising the crucial role of the tissue scale gradient of $\mathrm{H}_{2} \mathrm{O}_{2}$ for leukocyte attraction.

A study, also using zebrafish larvae, demonstrated that newly oncogene-transformed cells and their neighbours attracted leukocytes through $\mathrm{H}_{2} \mathrm{O}_{2}$ signalling. Utilising the $\mathrm{H}_{2} \mathrm{O}_{2}$-indicating dye, acetyl-pentafluorobenzene sulphonyl fluorescein, and 5,5-dimethyl-1-pyrroline $\mathrm{N}$-oxide (DMPO) that reports a history of ROS exposure, it was shown that $\mathrm{H}_{2} \mathrm{O}_{2}$ was stochastically and momentarily produced around V12RAS expressing cells in the epidermis. Like wounded epithelial cells, transformed cells generated $\mathrm{H}_{2} \mathrm{O}_{2}$ in a Duox dependent manner, highlighting parallels between oncogene-transformed cells and mechanical induced injury initiation of the host inflammatory response [32].

3.1.3. Hydrogen Peroxide as a Signalling Molecule in Inflammation. Functional roles of $\mathrm{H}_{2} \mathrm{O}_{2}$ during inflammation have been observed previously. Mechanistically, hydrogen peroxide can modulate protein function by reversible chemical modification of protein thiols, which can result in conformational changes affecting DNA binding, enzymatic activity, multimerisation, or protein complex formation. For example, the $\mathrm{NFkB} /$ Rel family, key regulatory molecules in the transcription of many genes involved in inflammation, is a well-known redox-sensitive transcription factor family [33]. $\mathrm{H}_{2} \mathrm{O}_{2}$-induced activation of $\mathrm{NFkB}$, which includes tyrosine phosphorylation of IkB and activation of IKK by $\mathrm{H}_{2} \mathrm{O}_{2}$ has been reported [34, 35]. Moreover, $\mathrm{H}_{2} \mathrm{O}_{2}$ can activate the release of high mobility group 1 protein from macrophages resulting in amplification of proinflammatory stimuli [36] or modulate leukocyte adhesion molecule expression and leukocyte endothelial adhesion [29]. VCAM1 , an endothelial scaffold on which leukocytes migrate, can activate signals in endothelial cells required for VCAM1-dependent leukocyte migration. Leukocyte binding to VCAM-1 stimulates NOX2 in endothelial cells, resulting in the generation of $\mathrm{H}_{2} \mathrm{O}_{2}$, which locally activates matrix metalloproteinases (MMPs). These MMPs in turn degrade matrix and endothelial cell surface receptors in cell junctions facilitating leukocyte transendothelial migration [37, 38].

These examples show how $\mathrm{H}_{2} \mathrm{O}_{2}$ can act as an intracellular or local signalling molecule but long-distance intercellular mechanisms of $\mathrm{H}_{2} \mathrm{O}_{2}$-mediated leukocyte recruitment were less well defined.
The open question of how leukocytes may receive the signal to initiate directional migration was recently addressed in another elegant study using the zebrafish model by Yoo et al. [39]. They have identified the SRC family kinase (SFK) Lyn as a redox sensor in neutrophils that detects hydrogen peroxide emanating from wounds and guiding their migration. Yoo and colleagues were able to provide direct evidence for punctate SFK activation at the leading edge of neutrophils in response to wounding. Through the knockdown of Duox, which is responsible for $\mathrm{H}_{2} \mathrm{O}_{2}$ production at the wound margin, they have explored the role of $\mathrm{H}_{2} \mathrm{O}_{2}$ in SFK activation. Duox knockdown prevented SFK phosphorylation indicating that activation of neutrophil SFKs may be dependent on the presence of hydrogen peroxide levels at wounds. Further evidence suggesting that SFKs can act as a redox sensor was provided by utilisation of SFK inhibitors that resulted in impairment of early neutrophil accumulation, while having no effect on epithelial hydrogen peroxide bursts [39].

Profiling SFK family members in zebrafish myeloid cells identified the Lyn kinase as a promising candidate acting as the redox sensor in neutrophils and macrophages. Morpholino knockdown of Lyn impaired directional migration of neutrophils to a tailfin wound in zebrafish larvae.

Further in vitro investigation revealed that hydrogen peroxide directly activates Lyn through the oxidation of Cys466, leading to downstream signalling, for example, Erk activation. This in vitro evidence was elegantly confirmed in vivo using a combination of genetic knockdown of Lyn and neutrophil-specific transgenic reconstitution of a Cys466 mutant or wild-type Lyn-GFP fusion.

In conclusion, these two sophisticated studies demonstrated a novel role of $\mathrm{H}_{2} \mathrm{O}_{2}$ as mediator of immediate inflammation and revealed aspects of the mechanisms resulting in leukocyte recruitment to a site of trauma (Figure 1). Evidence is accumulating that $\mathrm{H}_{2} \mathrm{O}_{2}$ signalling to phagocytes is a widely conserved mechanism present not only in zebrafish $[28,32,39]$ but also flies [31] and mammals $[39,40]$.

\section{Outlook}

The discovery of a new biological mechanism opens up a new line of research and poses numerous new questions to address. The most obvious being: How is Duox activated in epithelial cells upon wounding and how is the $\mathrm{H}_{2} \mathrm{O}_{2}$ gradient resolved? One hypothesis would place calcium as the immediate injury signal to the wounded cell in order to produce hydrogen peroxide through Duox. Physical disruption of plasma membranes results in an uncontrolled influx of calcium [41]. Giving credence to this hypothesis, evidence exists showing that DUOX activation by calcium regulates $\mathrm{H}_{2} \mathrm{O}_{2}$ generation [42].

In order to avoid excess tissue damage and persistent granulocyte recruitment/retention, the presence of the hydrogen peroxide gradient must be tightly regulated. Regulation could occur on the enzymatic level in terms of $\mathrm{H}_{2} \mathrm{O}_{2}$ production as well as on the molecular level in terms of $\mathrm{H}_{2} \mathrm{O}_{2}$ 


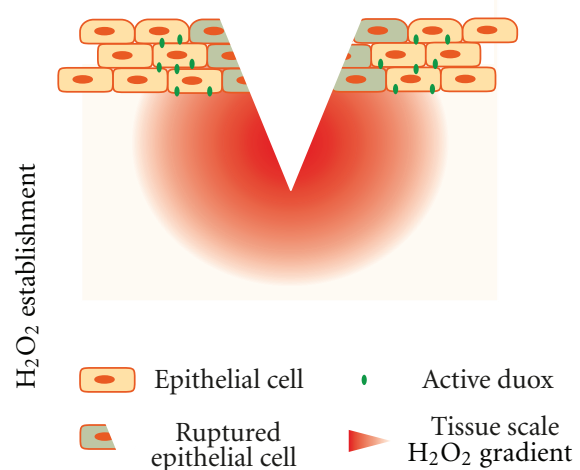

(a)

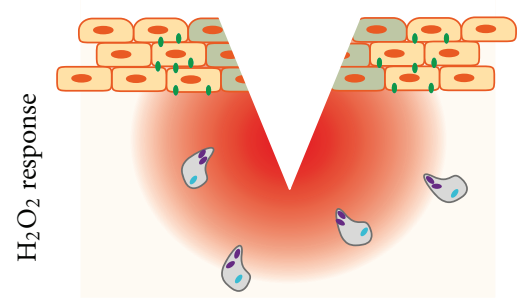

Migrating neutrophil

- Lyn

- pLyn

(c)

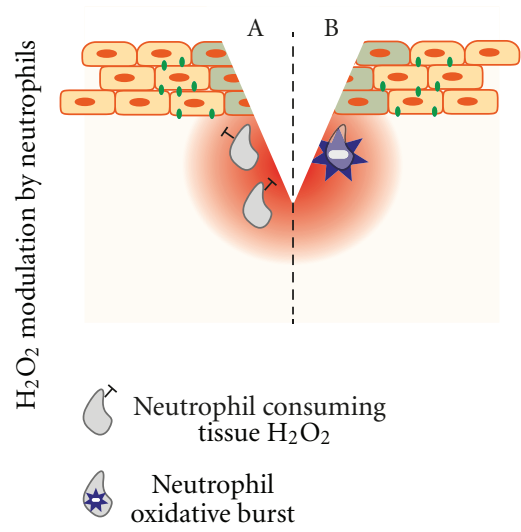

(e)

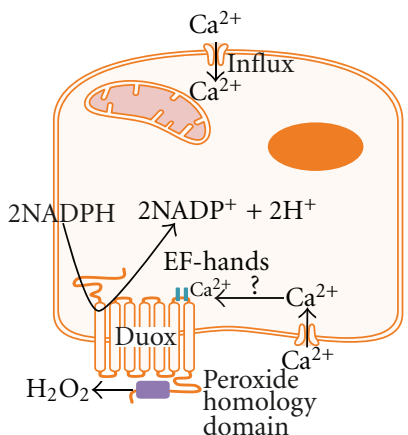

(b)

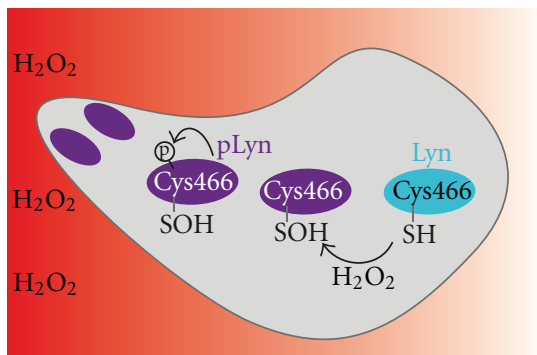

(d)

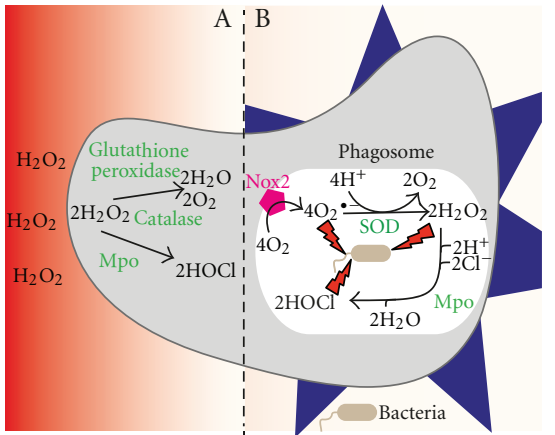

(f)

FIgURE 1: The role of hydrogen peroxide during the inflammatory response. (a) Upon tissue injury/trauma, epithelial cells adjacent to damaged cells activate the NADPH oxidase, Duox. Duox generates and establishes a localised tissue scale gradient of hydrogen peroxide, (b) Potential cellular events that result in Duox activation in epithelial cells. Disruption of epithelial cell membranes by mechanical trauma could lead to an increased influx of calcium in adjacent cells. Calcium binding to the EF-hand domain of Duox (residing in plasma membranes of epithelial cells), may initiate generation of hydrogen peroxide. (c) A tissue scale gradient of hydrogen peroxide acts as the first attraction signal for leukocytes. (d) Neutrophils sense hydrogen peroxide emanating from the wound partly through Lyn, a Src family kinase. Oxidation of Cys466 activates Lyn, resulting in autophosphorylation (pLyn) and punctate appearance of pLyn at the neutrophil leading edge is observed. (e) At the site of injury, neutrophils may alter hydrogen peroxide levels, both by consuming epithelial-derived hydrogen peroxide (A) or by local production of hydrogen peroxide through oxidative bursts (B). (f) Antioxidants, such as glutathione peroxidase and catalase could catalyse the decomposition of hydrogen peroxide into oxygen and water, while myeloperoxidase (Mpo) may consume hydrogen peroxide to produce hypochlorous acid (A). Neutrophils are equipped with multiple mechanisms to kill foreign organisms, one of them being the generation of ROS. Upon activation, phagosomal Nox2 generates superoxide, which is further converted into hydrogen peroxide by superoxide dismutase (SOD). Hydrogen peroxide alone and in conjunction with hypochlorous acid, generated by myeloperoxidase and other ROS exert bactericidal functions (B). 
stability. Oxidase activity results in membrane depolarisation due to the electrogenic properties of the enzymes to the point of NADPH oxidase inhibition. Prolonged $\mathrm{H}_{2} \mathrm{O}_{2}$ production depletes the NADPH pools, which may automatically result in cessation of $\mathrm{H}_{2} \mathrm{O}_{2}$ generation. Alternatively or in addition, neutrophil MPO could be responsible for the decrease in hydrogen peroxide levels upon arrival at the wound [24].

This mechanism suggests new approaches to therapeutically modulate both the onset of the cellular inflammatory response and its resolution, particularly as it involves a small, relatively unstable signalling molecule and is dependent on multiple enzymatic steps amenable to pharmacologic intervention.

\section{Authors' Contribution}

C. Wittman and P. Chockley contributed equally to this paper.

\section{Acknowledgments}

CW was supported by a PhD fellowship from the Helmholtz program "BioInterfaces international graduate school" (BIFIGS). Further support was provided by a KIT-RISC grant and by a Marie Curie International Reintegration Grant within the 7th European Community Framework Program (PIRG07-GA-2010-267552) to CG. The Australian Regenerative Medicine Institute is supported by grants from the State Government of Victoria and the Australian Government.

\section{References}

[1] J. Banchereau and R. M. Steinman, "Dendritic cells and the control of immunity," Nature, vol. 392, no. 6673, pp. 245-252, 1998.

[2] K. Hoebe, E. Janssen, and B. Beutler, "The interface between innate and adaptive immunity," Nature Immunology, vol. 5, no. 10, pp. 971-974, 2004.

[3] C. N. Serhan and J. Savill, "Resolution of inflammation: the beginning programs the end," Nature Immunology, vol. 6, no. 12, pp. 1191-1197, 2005.

[4] L. M. Coussens and Z. Werb, "Inflammation and cancer," $\mathrm{Na}$ ture, vol. 420, no. 6917, pp. 860-867, 2002.

[5] P. Libby, "Inflammation in atherosclerosis," Nature, vol. 420, no. 6917, pp. 868-874, 2002.

[6] C. L. Van Hove, T. Maes, G. F. Joos, and K. G. Tournoy, "Chronic inflammation in asthma: a contest of persistence vs resolution," Allergy, vol. 63, no. 9, pp. 1095-1109, 2008.

[7] K. Bedard and K. H. Krause, "The NOX family of ROS-generating NADPH oxidases: physiology and pathophysiology," Physiological Reviews, vol. 87, no. 1, pp. 245-313, 2007.

[8] M. Rojkind, J. A. Domínguez-Rosales, N. Nieto, and P. Greenwel, "Role of hydrogen peroxide and oxidative stress in healing responses," Cellular and Molecular Life Sciences, vol. 59, no. 11, pp. 1872-1891, 2002.

[9] C. A. Pritsos, "Cellular distribution, metabolism and regulation of the xanthine oxidoreductase enzyme system," ChemicoBiological Interactions, vol. 129, no. 1-2, pp. 195-208, 2000.
[10] A. T. Demiryürek and R. M. Wadsworth, "Superoxide in the pulmonary circulation," Pharmacology and Therapeutics, vol. 84, no. 3, pp. 355-365, 1999.

[11] B. T. Kawahara, M. T. Quinn, and J. D. Lambeth, "Molecular evolution of the reactive oxygen-generating NADPH oxidase (Nox/Duox) family of enzymes," BMC Evolutionary Biology, vol. 7, article 109, 2007.

[12] B. Rada and T. Leto, "Oxidative innate immune defenses by Nox/Duox Family NADPH oxidases," Contributions to Microbiology, vol. 15, pp. 164-187, 2008.

[13] N. Driessens, S. Versteyhe, C. Ghaddhab et al., "Hydrogen peroxide induces DNA single- and double-strand breaks in thyroid cells and is therefore a potential mutagen for this organ," Endocrine-Related Cancer, vol. 16, no. 3, pp. 845-856, 2009.

[14] T. L. Leto and M. Geiszt, "Role of Nox family NADPH oxidases in host defense," Antioxidants and Redox Signaling, vol. 8, no. 9-10, pp. 1549-1561, 2006.

[15] E. Owusu-Ansah, A. Yavari, S. Mandal, and U. Banerjee, "Distinct mitochondrial retrograde signals control the G1-S cell cycle checkpoint," Nature Genetics, vol. 40, no. 3, pp. 356-361, 2008.

[16] E. Owusu-Ansah and U. Banerjee, "Reactive oxygen species prime Drosophila haematopoietic progenitors for differentiation," Nature, vol. 461, no. 7263, pp. 537-541, 2009.

[17] J. Li, M. Stouffs, L. Serrander et al., "The NADPH oxidase NOX4 drives cardiac differentiation: role in regulating cardiac transcription factors and MAP kinase activation," Molecular Biology of the Cell, vol. 17, no. 9, pp. 3978-3988, 2006.

[18] S. Lange, J. Heger, G. Euler, M. Wartenberg, H. M. Piper, and H. Sauer, "Platelet-derived growth factor BB stimulates vasculogenesis of embryonic stem cell-derived endothelial cells by calcium-mediated generation of reactive oxygen species," Cardiovascular Research, vol. 81, no. 1, pp. 159-168, 2009.

[19] R. Colavitti, G. Pani, B. Bedogni et al., "Reactive oxygen species as downstream mediators of angiogenic signaling by vascular endothelial growth factor receptor-2/KDR," Journal of Biological Chemistry, vol. 277, no. 5, pp. 3101-3108, 2002.

[20] K. Hensley, K. A. Robinson, S. P. Gabbita, S. Salsman, and R. A. Floyd, "Reactive oxygen species, cell signaling, and cell injury," Free Radical Biology and Medicine, vol. 28, no. 10, pp. 14561462, 2000.

[21] S. P. Gabbita, K. A. Robinson, C. A. Stewart, R. A. Floyd, and K. Hensley, "Redox regulatory mechanisms of cellular signal transduction," Archives of Biochemistry and Biophysics, vol. 376, no. 1, pp. 1-13, 2000.

[22] W. M. Nauseef, "Assembly of the phagocyte NADPH oxidase," Histochemistry and Cell Biology, vol. 122, no. 4, pp. 277-291, 2004.

[23] W. M. Nauseef, "Contributions of myeloperoxidase to proinflammatory events: more than an antimicrobial system," International Journal of Hematology, vol. 74, no. 2, pp. 125-133, 2001.

[24] S. J. Klebanoff, "Myeloperoxidase: friend and foe," Journal of Leukocyte Biology, vol. 77, no. 5, pp. 598-625, 2005.

[25] L. Pase, C. J. Nowell, and G. J. Lieschke, "In vivo real-time visualization of leukocytes and intracellular hydrogen peroxide levels during a zebrafish acute inflammation assay," Methods in Enzymology, vol. 506, pp. 135-156, 2012.

[26] G. Y. Chen and G. Nuñez, "Sterile inflammation: sensing and reacting to damage," Nature Reviews Immunology, vol. 10, no. 12, pp. 826-837, 2010.

[27] O. Takeuchi and S. Akira, "Pattern recognition receptors and inflammation,” Cell, vol. 140, no. 6, pp. 805-820, 2010. 
[28] P. Niethammer, C. Grabher, A. T. Look, and T. J. Mitchison, "A tissue-scale gradient of hydrogen peroxide mediates rapid wound detection in zebrafish," Nature, vol. 459, no. 7249, pp. 996-999, 2009.

[29] C. K. Sen and S. Roy, "Redox signals in wound healing," Biochimica et Biophysica Acta, vol. 1780, no. 11, pp. 1348-1361, 2008.

[30] V. V. Belousov, A. F. Fradkov, K. A. Lukyanov et al., "Genetically encoded fluorescent indicator for intracellular hydrogen peroxide," Nature Methods, vol. 3, no. 4, pp. 281-286, 2006.

[31] S. Moreira, B. Stramer, I. Evans, W. Wood, and P. Martin, "Prioritization of competing damage and developmental signals by migrating macrophages in the Drosophila embryo," Current Biology, vol. 20, no. 5, pp. 464-470, 2010.

[32] Y. Feng, C. Santoriello, M. Mione, A. Hurlstone, and P. Martin, "Live imaging of innate immune cell sensing of transformed cells in zebrafish larvae: parallels between tumor initiation and wound inflammation," PLoS Biology, vol. 8, no. 12, Article ID e1000562, 2010.

[33] H. Jay Forman and M. Torres, "Redox signaling in macrophages," Molecular Aspects of Medicine, vol. 22, no. 4-5, pp. 189-216, 2001.

[34] S. Schoonbroodt, V. Ferreira, M. Best-Belpomme et al., "Crucial role of the amino-terminal tyrosine residue 42 and the carboxyl- terminal PEST domain of $\mathrm{I} \kappa \mathrm{B} \alpha$ in NF- $\kappa \mathrm{B}$ activation by an oxidative stress," Journal of Immunology, vol. 164, no. 8, pp. 4292-4300, 2000.

[35] Z. Yin, V. N. Ivanov, H. Habelhah, K. Tew, and Z. Ronai, "Glutathione S-Transferase p elicits protection against $\mathrm{H}_{2} \mathrm{O}_{2}$ induced cell death via coordinated regulation of stress kinases," Cancer Research, vol. 60, no. 15, pp. 4053-4057, 2000.

[36] D. Tang, Y. Shi, R. Kang et al., "Hydrogen peroxide stimulates macrophages and monocytes to actively release HMGB1," Journal of Leukocyte Biology, vol. 81, no. 3, pp. 741-747, 2007.

[37] J. S. Alexander and J. W. Elrod, "Extracellular matrix, junctional integrity and matrix metalloproteinase interactions in endothelial permeability regulation," Journal of Anatomy, vol. 200, no. 6, pp. 561-574, 2002.

[38] J. M. Cook-Mills, "Hydrogen peroxide activation of endothelial cell-associated MMPS during VCAM-1-dependent leukocyte migration," Cellular and Molecular Biology, vol. 52, no. 4, pp. 8-16, 2006.

[39] S. K. Yoo, T. W. Starnes, Q. Deng, and A. Huttenlocher, "Lyn is a redox sensor that mediates leukocyte wound attraction in vivo," Nature, vol. 480, pp. 109-112, 2011.

[40] I. V. Klyubin, K. M. Kirpichnikova, and I. A. Gamaley, "Hydrogen peroxide-induced chemotaxis of mouse peritoneal neutrophils," European Journal of Cell Biology, vol. 70, no. 4, pp. 347-351, 1996.

[41] A. Draeger, K. Monastyrskaya, and E. B. Babiychuk, "Plasma membrane repair and cellular damage control: the annexin survival kit," Biochemical Pharmacology, vol. 81, no. 6, pp. 703-712, 2011.

[42] S. Rigutto, C. Hoste, H. Grasberger et al., "Activation of dual oxidases Duox1 and Duox2: differential regulation mediated by cAMP-dependent protein kinase and protein kinase Cdependent phosphorylation," Journal of Biological Chemistry, vol. 284, no. 11, pp. 6725-6734, 2009. 


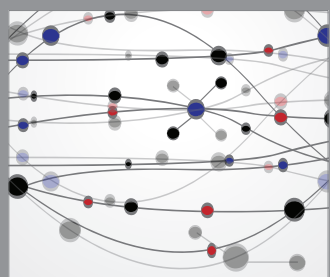

The Scientific World Journal
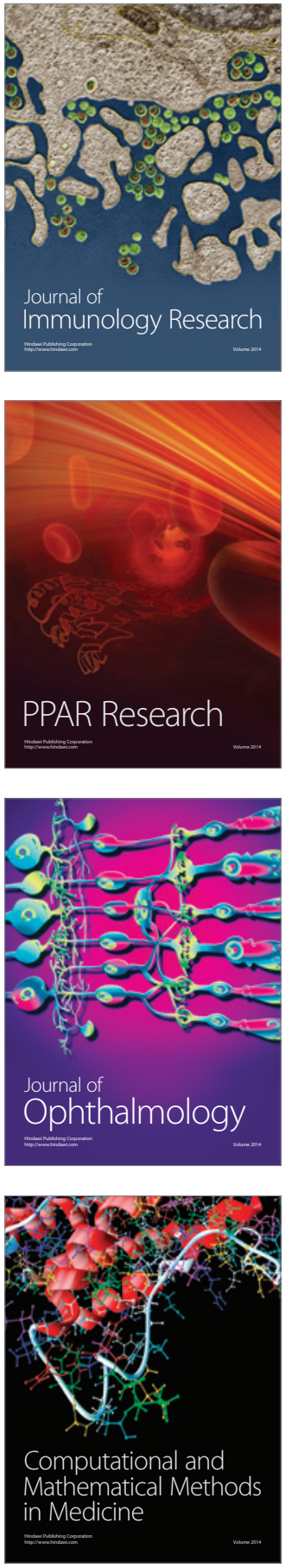

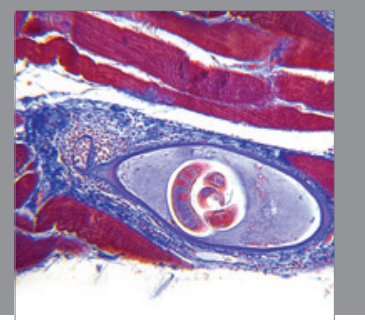

Gastroenterology

Research and Practice
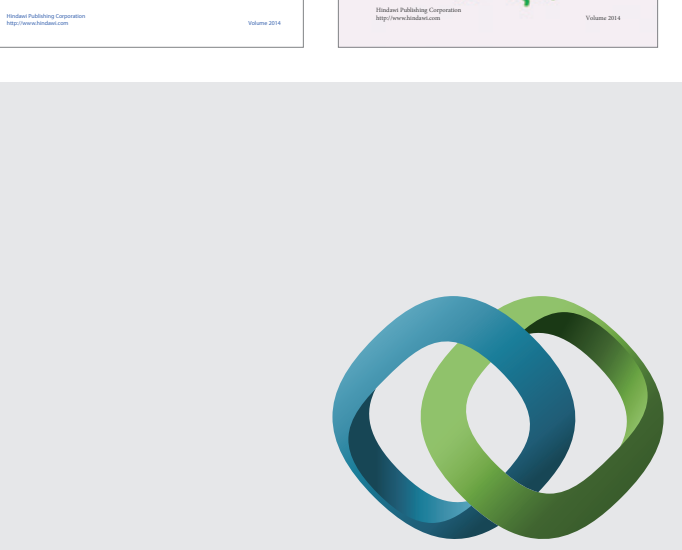

\section{Hindawi}

Submit your manuscripts at

http://www.hindawi.com
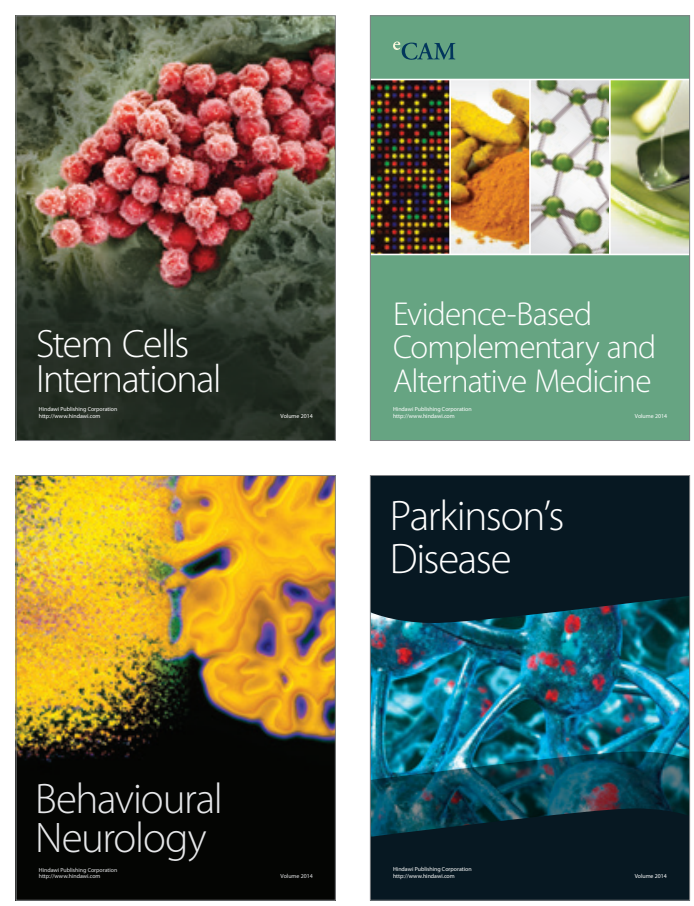

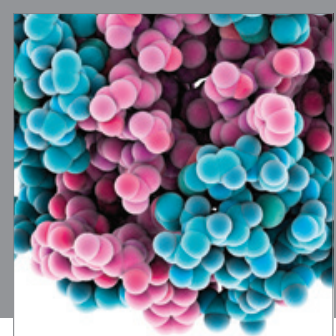

Journal of
Diabetes Research

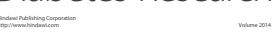

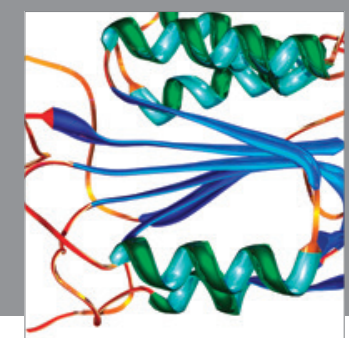

Disease Markers
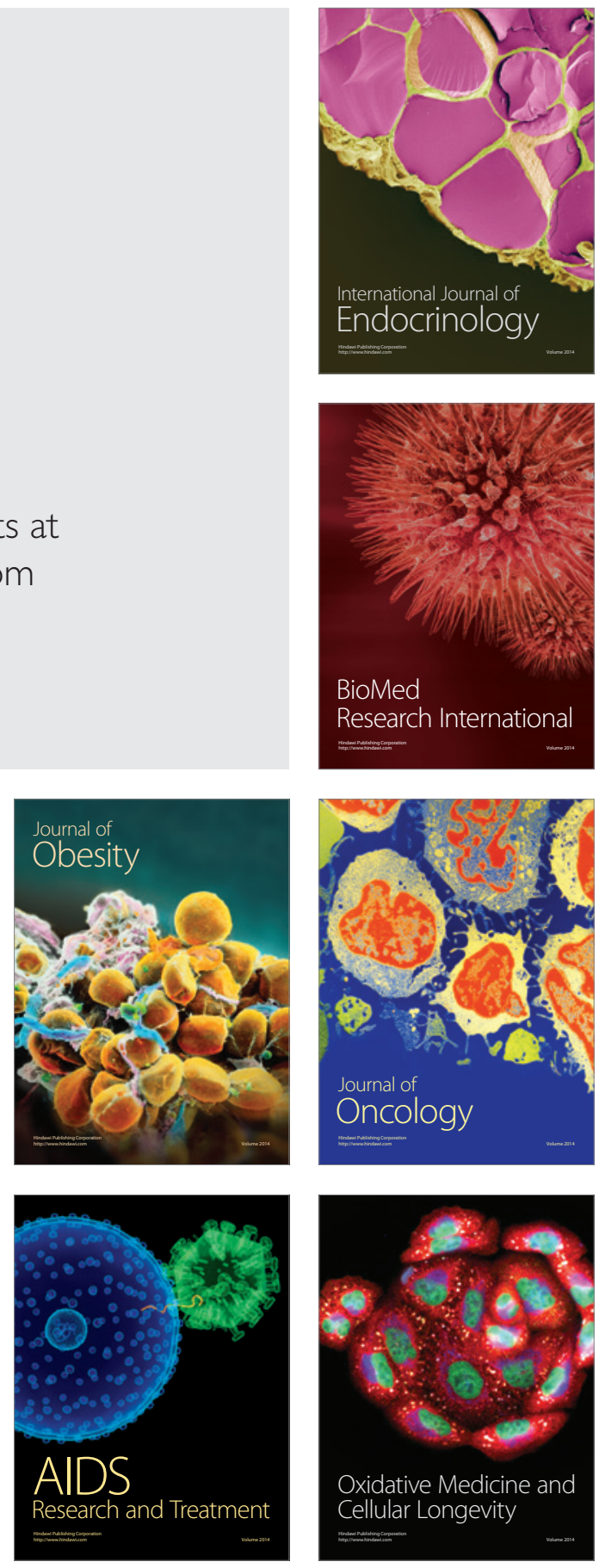